\title{
A Survey on Biomedical Informatics for Computer-Aided Decision Support System
}

\author{
${ }^{1}$ Meilin Gray \\ ${ }^{1}$ School of Public Health, Sun Yat-sen University, Guangdong Province, China \\ ${ }^{1}$ Gray22554441@hotmail.com
}

\author{
Article Info \\ Journal of Biomedical and Sustainable Healthcare Applications (http://anapub.co.ke/journals/jbsha/jbsha.html) \\ Doi: https://doi.org/10.53759/0088/JBSHA202101011 \\ Received 10 October 2020; Revised form 25 December 2020; Accepted 06 April 2021. \\ Available online 05 July 2021. \\ (C)2021 Published by AnaPub Publications.
}

\begin{abstract}
Biomedical computing for computer-aided biomedical diagnostics and the decision support system has developed a platform for the biomedical setting during the last few decades. As early as 1971, there were elaborate and basic applications of management information systems driven by biomedical informatics. According to a 1994 assessment, this field's literature stretches back to the 1950s. Medical decision is more challenging than ever for doctors and other caregivers due to the amount and complexity of contemporary patient information. This circumstance necessitates the application of medical computing technologies to evaluate data and formulate suggestions and/or forecasts to aid decision makers. Over the past two decades, healthcare informatics tools, such as computer-aided decision support, have grown indispensable and extensively employed. This article gives a quick overview of such technologies, their productivity applications and methodology, as well as the problems and directions they imply for the future.
\end{abstract}

Keywords - Clinical Decision Support System (CDSS), Biomedical Computing, Computer-Assisted Medical Diagnosis

\section{INTRODUCTION}

Advancements in biomedical signals and image collecting technology have resulted in an increase in the intricacy of health data acquired. Aside from the fact that medical data is intrinsically more complicated, the amount of data acquired per patient is continually increasing. Healthcare systems and high-throughput measurement methods already create hundreds of photos and vast quantities of datasets for every client in couple of minutes, making it problematic for healthcare practitioners to sort through the dataset and provide prompt diagnosis and progression. In healthcare, there is now a major demand for improvement and advancement of computer-aided management information systems, with further growth projected. Clinical applications of medical bioinformatics methodologies in the type of computer-oriented management information systems may be traced back to 1971, when Dombal's AAP-help program, created at Leeds University, focused on automating definitive diagnostics discomfort of the abdomen. A rule-based integration framework called INTERNIST-I was created in 1974 to assist in the identification of complicated medical issues in interior medicine. Among several computer-based diagnostics decisions systems and methods, these constitute significant advancements in early healthcare informatics applications. There has been a significant change since their introduction, with widespread recognition of their effectiveness in increasing practitioner care quality and patient results.

There have been comparative investigations on related themes as a result of the vast research done in the domain Medical interventions are discussed in broad terms, as well as future research directions. Rzevski [1] also describe the evolution of computer-aided prognostic systems that use multiresolution, heterogeneous healthcare data to forecast patient and illness outcomes. Between 1954 and 1993, Miller published an essay in which he compiled a thorough summary of major diagnostic and decision-making research. Pearson et al., in a more recent paper, conducted a comprehensive evaluation of computerised clinical decision - making from 1990 to 2007. There are 56 studies evaluated in this paper, with 38 of them focusing on technologies used in treatment beginning, 23 on computer-based healthcare management throughout therapy, and three on circumstances for therapeutic discontinuation. The authors conclude that the computerbased structures that start recommendation to fine-tune conventional therapy by infection prevention and control, making adjustments the dosage and length forms of prescription medications, or rising diagnostic test prognosis in patients on long-term therapeutic interventions are most affective. 
Previous research has also shown more particular subgenres of healthcare computing methodologies and their applications as computer-aided diagnostic systems. Scientists talk about systems that give clinical interpretations based on picture texture classification and the effects of management information systems in clinical radiology. This article presents a broad overview of biomedical informatics technologies and approaches which have been structured as CDSSs, as well as the issues that have arisen as a consequence of these implementations, such as system testing and end-user acceptance. This is how the paper is structured: Section II presents the characteristics evaluation of CDSS. Section III focuses on its application. Section IV focuses on the methodology segment. Section V presents an analysis of the Computer-Aided Decisions in Bioinformatics. Section VI presents an analysis of the adoption issues. Section VII presents the authentication and standards for success. Lastly, Section VIII concludes the paper and presents directions for future research.

\section{CHARACTERISTICS}

Clinical Decision Support Systems (CDSSs) are an intensive data technology that produces advice about a health situation using elements from patient information. This means that CDSSs are typically systems for decision support, which uses knowledge administration to provide clinical guidance for acute management depending on various pieces of patient information.

\section{Purpose}

Contemporary CDSS are primarily designed to aid doctors during the period of healthcare. This shows that practitioners operate with CDSSs to evaluate the patient datasets and reach at a diagnostics agreement. CDSS was initially considered as being applied to make biomedical decisions for the healthcare practitioners. The practitioners could compute datasets and wait for the CDSSs to generate the most effective option that practitioners would therefore work on. Nonetheless, the current practices of applying CDSS to aid in highlighting that the practitioners collaborate with CDSSs draw on their expertise and that CDSSs to provide an effective evaluation to the patients' data compared to either the human brain or CDSSs could generate their own. CDSSs normally provides recommendations for the physician to consider, and the doctor is required to extract crucial data from the supplied findings while dismissing inaccurate CDSS recommendations.

\section{Types}

CDSSs are categorized into group: Knowledge-based and non-knowledge-based:

Diagnosis Decision Support Systems (DDSSs) is one example of how a physician may utilize a CDSS. A DDSS asks for some of patients' information and then suggests a set of diagnostics. The clinician then analyzes the DDSS results to see which treatments are applicable and which aren't, and, if required, performs more procedures to pinpoint the diagnoses. A case-based reasoning (CBR) technology is another form of a CDSS. Relevant previous information might be used by a CBR system to assist identify the proper number of beams and ideal beam orientations to be used in radiation for patients with brain cancer; healthcare physicist and physicians would then assess the suggested treatment regimen to see whether it was viable.

The time of the usage of a CDSS is also another key category. Clinicians utilize these technologies at the point of care to assist them when treating a patient, with the usage occurring either before, during, or after the diagnosis. Pre-diagnosis CDSS technologies are used to assist doctors in making diagnoses. The CDSS is utilized during diagnosis to assist the physician analyze and filter their exploratory diagnostics options in order to enhance their final outcomes. Post-diagnosis CDSS technologies extract data to attract connection between patients and biomedical histories, as well as clinical studies, in order to forecast future occurrences. Decision assistance will substitute physicians in typical activities in the future, according to predictions made in 2012.

Another option is to use a DDSS, which was previously managed by the patient but is now operated by a nonmedically trained phone operator, to triage medical emergencies out of minutes by proposing an appropriate upcoming phase to individuals, such as requesting ambulance or seeing a biomedical expert the following day. An advice is centred on the prevailing fact and an implied opinion concerning what the worst case diagnosis is projected to be that it might have dismissed by the patient or cellular operators if prudence advises or common sense differently. It is not usually disclosed to the patient since it may be inaccurate and is not dependent on the judgment of a medically competent individual; it should only be used for first screening.

\section{Knowledge-based CDSSs}

The knowledge base, the inference system, and the communication mechanism are the three main components of most CDSSs. The guidelines as well as affiliations of executable data are stored in the body of knowledge, which are usually 
expressed as IF-THEN guidelines. If this were a drug interaction detection method, a guideline could be that if narcotic $\mathrm{X}$ AND narcotic $\mathrm{Y}$ are both taken, the user should be notified. A high tech user might edit the base of knowledge using a different system in order to keep it current with new drugs. The knowledge base's guidelines are combined with the patients' data by inference approach. The systems' interaction methods allowed it to demonstrate the consumer the outcomes as well as receive input.

Knowledge objects must be expressed in a computable manner using an interpretation dialect like GELLO and CQL (Clinical Quality Language). For instance -, in case the patients have type 2 diabetes and haemoglobin A1c results of the test is much less than $8 \%$, re-testing should be done if it has been more than four months, but re-testing should be done if it has been more than three months. The Clinical Quality Language is the primary priority of a HL7CDS WG (CQL). CMS has stated that it will specify eCQMs using CQL.

\section{Non-knowledge-based CDSSs}

Machine learning helps machines to understand from previous experiences and/or detect patterns in clinical evidence in CDSSs that do not employ a knowledge and understanding. Writing guidelines and obtaining expert advice are no longer required. For issues of dependability and responsibility, most practitioners do not employ machine learning algorithms directly for diagnostics since they cannot understand the logic for their results. They may, however, be valuable as postdiagnostic algorithms, pointing up trends for physicians to investigate further. Support Vector Machine (SVM) and Artificial Neural Networks (ANNs) and genetic algorithms are the three types of non-knowledge base system as of 2012.

- ANNs evaluate the trends of patients' data and effectively develop the connections between different symptoms and the diagnosis by utilizing the node and weighted linkage between them.

- To generate the most effective CDSSs results, evolutionary computations apply evolutionary transitions with focussed selections. Elements of random collection of remedies of complex issues are assessed using the selected algorithm. The winning remedies are modified and recombined, and the procedure is repetitive. This repeats itself until the best answer is identified. They are compared to the NNs (Neural Networks) in that they are black boxes, which focus on making sense of patients' data.

- In contrast to the knowledge-based method, which covers the evaluation of many distinct illnesses, nonknowledge-based networkings sometimes concentrate on a small variety of symptoms, such as signs of a specific disease.

\section{Effectiveness}

The data on CDSS's efficacy is contradictory. CDSS has a greater impact on certain pathological conditions than on others. Sugar level monitoring, blood donation strategic planning, physiologic degradation preventative medicine, pressure ulcer preventative measures, chronic kidney preventive care, and venous thromboembolism prophylaxis were all identified as six health conditions in which CDSS enhanced clinical outcomes in healthcare setting in a 2018 review of the literature. When the CDSS and the electronic medical record were merged in 2014, a comprehensive review found no advantage in terms of risk of mortality. Nevertheless, in regard to other outcomes, there might be some advantages. CDSSs enhanced practitioner efficiency in $64 \%$ of trials and health experience in 13 percentage points of studies, according to a comprehensive analysis published in 2005. Electronically controlled cues, rather than needing user engagement of the system, are one of the CDSS characteristics linked to increased practitioner efficiency. Decision assistance systems enhanced medical care in 68 percent of studies, according to a 2005 systematic review. Integration into the healthcare setting rather than as a distinct log-in or display, digital instead of paper-based template, decision assistance at the time and place of care rather than before to it, and care suggestions were among the CDSS elements related with effectiveness. Subsequently assessments, nevertheless, were less enthusiastic about CDS's impacts, with one from 2011 asserting that "there is a massive disparity between the speculated and clearly proven benefits of [Clinical decision support and other] e healthcare innovations... their cost-efficiency has yet to be illustrated." In 2014, Ciapponi [2] released the first long-term research of a CDSS, which looked at its efficacy in adopting sensible management of infectious diseases over a 5-year period.

\section{APPLICATIONS}

CDSS have been devised and used in a variety of medical application domains. The following sections go through some of the most important applicability domains. 


\section{Radiology}

Computer-assisted image processing has been a hot topic in this field. For clinical applications, combining visualizations, image analysis, and computer vision for decision-making has shown to be beneficial. Numerous biomedical computing approaches have been developed for application-specific applications using several biomedical imaging approaches such as Magnetic Resonance Imaging (MRI), X-rays, Computed Tomography (CT), and Functional MRI (FMRI). One of the researches examines more than 150 papers on computer-aided diagnostics in computed tomography that were published prior to 2001. The continuous enthusiasm in computer-aided diagnostics for computed tomography is reflected in the results of this study. Several researches have also been conducted on the developments of decisions support systems centered on CT detection analysis. Among these is the project purposed at structuring the computer-aided diagnostic system that evaluates the brain CT images of the patients with Traumatic Brain Injuries (TBIs) automatically. The intracranial pressure (ICP) inside the brain is also automatically estimated by the device. Ji, Smith, Huynh and Najarian [3] also describe the nature of computer-aided decision-making approaches for the pelvis injury in another research. Researchers provide an automatic methodology for evaluating the dimension from CT scan of patients with pelvis injuries. The authors present a review of the design and functioning of radiographic management information systems, as well as instances of their growth and progress over the past decades.

\section{Emergency Medication and Intensive Care Unit (ICU)}

In the area of biomedical computing and management information systems, medical technology is among the most prominent research areas. It is vital to give evaluation and management to individuals in the ICU (Intensive Care Unit) and the emergency departments as soon as possible. Because intensive care facilities are often overburdened, it is essential to handle and distribute resources to fundamentally ill individuals who necessitate them ultimately. CDSSs are crucial in reducing diagnostic time, increasing allocation of resources effectiveness, and lowering clinical outcomes. A study comparing computer-assisted decision-making methods for severe injuries is described by the scientists. Case-based argumentation strategies for estimating clinical outcomes and resource consumption indicate how case-based argumentation approaches may drastically enhance patient care in ICUs. Pinheiro, Candore, Zaccaria, Slattery and Arlett [4] describe a CDSS that incorporates both case-oriented and rule-oriented and operates effectively with generated and actual ICU datasets. A computer amber alert intended to detect adverse drug events in medical settings is described by the authors. This technology is said to be able to send out notifications to patients who are at a higher risk of using AEDs. According to the research, 265 (45\%) of the 595 correct positive warnings were misunderstood by doctors prior towards the alert notice, indicating that the system has a lot of potential for continual patient monitoring systems.

\section{Cardiovascular Medication}

Intermittent or therapeutic cardiovascular signals surveillance for detecting diseases or forecasting imminent cardiovascular events might be a very important tool. Several medical computing research initiatives are under underway to provide computer-aided options for various parts of cardiovascular medicine. Authors in [5] developed a computeraided diagnostic method that analyzes patients' electrocardiograph (ECG) data and automatically detects and classifies arrhythmias. Within the data utilized, the authors state 100 percent accuracy in categorization. Arknæs-Pedersen [6] looked at a number of researches that analyze the heart's auscultation signal and give diagnostic decision assistance to doctors. The authors express their ideas on the innovation and implementation of a nonlinear approach for analyzing dysrhythmias employing ECG data in order to forecast defibrillation effectiveness with high yields precision. The PetCO2 signal was also included in the research, which significantly improved the prediction models' resilience.

\section{Dental Medication}

The discipline of orthodontics has benefited greatly from computerized clinical diagnostic and decision support tools. Sánchez-Pi, Carbó and Molina [7] present a knowledge-based system that performed imaging techniques on plain radiographs as a CDSS for observer effectiveness. This research used 102 approximal surface radiography pictures and 16 specialist doctors to determine if caries was present and whether treatment was necessary. According to the study, dental professionals who utilized the method to generate diagnoses saw a substantial improvement in their capacity to accurately identify caries, as well as a greater average diagnostic performance and suggestion for restoration of discovered calcification surfaces. Kositbowornchai, Siriteptawee, Plermkamon, Bureerat and Chetchotsak [8] also suggest a computer-assisted caries detection method based on image analysis of intraoral different cameras. The viability of 
employing modern image processing and artificial intelligence approaches to detect caries in digital photographs is discussed in this work.

\section{Cancer}

In cancer diagnosis and therapy, biomedical computing has started to play a significant role. A comprehensive review of numerous studies in the area of cancer decision-making instruments is offered in a study undertaken by academics. The review provides an overview on artificial neural network research in particular. The article finds that 21 of the 27 studies, all of which were diagnostic tests or controlled trials, showed therapeutic benefits, whereas the other six did not. Another research analyzed merging contradictory data from several modalities to enhance computer-aided decision-making for diagnosis of cancer. According to the scientists, their suggested approach surpasses two prominent machine-learning methods, linear regression techniques or convolutional neural networks, sometimes. Four distinct computer-aided assistance systems for tumor prediction and diagnosis are briefly discussed in a research released. The first method is an image-priented risk score method that generates digitized biopsies to predict the fate of the oestrogen biomarker for cancer patients. The second technique, which is explained in detail in the article chapters, uses digital histology to estimate the level of lymphocytic infiltration. Patients with different Gleason grade of hepatocellular carcinoma may be distinguished from percutaneous tissue biopsies using the third approach presented. In order to identify among higher and lower patients with prostatic cancer recurrence after surgical resection, the final system combines quantifiable image characteristics derived from digitised histopathological with protein expression measures acquired from spectrometry.

Using computer-aided diagnostic tools, Moinet, Mandil and Serre [9] were able to reduce interobserver variability in mammography interpretation. According to the authors, adopting computer-aided diagnosis instruments for mammography interpretations has the capacity to effectively minimize the diversity among professional judgments while also improving diagnostic accuracy. The researchers also review and compare the approaches employed in different augmentation and recognition techniques in another research. This also includes the separation of mammographic features, classifications, and their effectiveness in detecting and classifying microcalcification groups. The optimization methodology for establishing case-oriented computer-assisted objective to create a platform for mammography scanning is described in a publication by the authors. According to the research, the suggested strategy enhances the overall effectiveness and recognition rates of these computers by a substantial amount. The article shows how the researchers used gene levels to classify cancer subgroups and forecast prognosis in diffuse large B-cell lymphoma (DLBCL). Computer-aided breast cancer diagnosis systems have gained enhanced techniques, according to research by specialists. The study introduces novel detection strategies for detecting subtle symptoms of breast cancer, like localized structural distortion and universal contralateral asymmetrical, which are difficult to spot.

\section{Pediatric Medication}

In prenatal and pediatrics care facilities, computer-aided diagnostic and CDSS have gained popularity for a number of uses. With the help of a study case of a web-based paediatric differentiated screening tool, Azami [10] examine the possibilities of diagnostics and CDSS in pediatric contexts. Segev [11] detail the different uses of such diagnostic assistance systems and their future research goals in a separate publication. Atabakhsh [12] describes an artificial intelligence-based approach for predicting medical results and resource consumption that use case-based argumentation. The report describes how a system like this was originally designed for adult ICUs but was later adapted to work in newborn ICUs. The findings of a brief clinical pilot research conducted in the newborn ICU were highly positive, according to the publication, and clinicians were particularly interested in their prospective therapeutic use. Tan et al. released a review study on newborn care CDSS. The goal of this study was to examine whether using CDSS had any influence on the death and morbidity rates of newborn newborns, as well as if clinicians treating these babies performed better. The authors also conducted an assessment of decision-making mechanisms in pediatric critical care settings. Liability, human - system, auditing, architecture, and alarm fatigue are all discussed in the study as aspects to consider when deploying such technologies in pediatric offices. The research concludes that choosing and deploying such technologies in clinical settings needs significant caution, but when done properly, it offers a lot of promise for benefitting and enhancing medical care in pediatric critical care settings.

\section{METHODOLOGY}

The development of health informatics and computer-assisted diagnostic assistance systems employs a number of core computational approaches. The approaches and methods used are determined by the application domains and performance indicators that are needed. The next sections go through some of the key characteristics of such systems. 


\section{Knowledgeable Schemes, Case-Based Cognitive, and Rule-Based Schemes}

Knowledge bases, rule-based structures (fuzzy or crisp), and case-based logic are all based on the information gathered by professionals in a particular discipline. Perspectives, diagnostics, and progression, among other elements, are combined into rule-based analytical structures, from which particular diagnostic ideas are produced. Campanella and Ribeiro [13] proposed a dynamic decision - making framework that was developed using both case-oriented and rule-b reasoning and is used in ICUs to assist clinicians in setting priorities. Bagheri and Mardaneh discuss a fuzzy logic technique to computeraided diagnostic medical tools for clinical environments in another work. Professional data is gathered into fuzzy representations and logical frameworks in this approach to determine a disease phase utilizing temporal dependencies in symptoms duration.

\section{Signal and Image Processing}

When producing diagnostic recommendations, several computerized diagnostic assistance systems analyze a range of patient data. The systems screen raw outputs and images to effectively extract fundamental features and patterns, which are then used to calculate diagnostics and decisions support information data and provide it to doctors. The signal processing platform is presented to examine ECGs to categorize instances of arrhythmia in patients. Cockx, Denolf, Vanhoof and. Stahl [15] created a signal transaction processing that determines the best moment to defibrillate patients who are experiencing cardiac event or ventricular tachycardia. The authors suggest an image processing method that analyzes CT scans of individuals with pelvic fractures to provide a qualitative evaluation of observed hemorrhage. The authors work on establishing a computer-assisted fractures detection method uses many CT scans of individuals with pelvic injuries to locate and quantify possible injuries.

\section{Machine Learning $(M L)$}

More complicated and complex medical computing technologies are being built as a result of ongoing advances in the area of cognitive computing. Machine learning-based systems that can predict and categorize illnesses are primarily based on this technology. There are no optimal ML approaches, which could be utilized to mitigate the various learning problems; rather, the most effective methodologies are identified by the design. One paper, for instance, presents a thorough assessment of neural network models in cancer diagnostic and treatment CDSS. In a research employing cancer datasets, Malla [16] show how a tailored machine learning approach outperforms established techniques like neural networks and support vector regression. For traumatic damage assessment, Yang, Nazir, Xu and Ali [7] examine a number of data mining algorithms used in decision-making algorithms.

\section{COMPUTER-AIDED DECISIONS IN BIOINFORMATICS}

Bioinformatics has developed as a dynamic and quickly developing discipline during the past two decades. However, given the antiquity of the subject, the pluralities of computer-aided management information systems provide representations of medical computing systems. As a result, only a small percentage of presently employed computer-aided management information systems those are dependent on informatics methodologies. Zimmerman and Rothmeier [18] compared the history, basic underpinnings, and scientific methodologies of the two complimentary but distinct areas of medical computing and informatics in a research. The authors discuss how informatics expertise may help with medical software development. Future research that combines both computing subdisciplines is critical, according to the authors, for achieving major advancements in clinical settings and biomedical sciences.

Integrating the multi-modal datasets; and integrating bioinformatics and medical informatics has typically indicated considerable potentials. Authors in [19] present an analysis on computer-assisted diagnostic and prognostic systems, which utilize multiple modal data, incorporating computerize evaluation of images and computerized patients data e.g. the tissues and genetic data for forecasting survival and outcomes, as previously noted. These initiatives diagnose and provide prognoses for cancer patients by analyzing protein definition and other sets of data utilizing standardized bioinformatics methodologies. The researchers utilized the time series micro-array gene expression profile data to forecast how individuals will react to pegylated interferon therapy and reported their findings. In virus research, computer-aided decision-making techniques that have been modified with bioinformatics knowledge have started to have a favorable influence. The authors propose a computational strategy for discovering the underlying approaches of disease resistance in particular individuals centered on the expressions of gene patterns and networking evaluation of the virus and host interactive networking in a publication. Another research uses a network-based analysis to present a unique method for identifying liver cirrhosis and hepatocellular disorders. 


\section{ADOPTION ISSUES}

\section{Clinical Issues}

The quest to merge multidimensional data with computational biology and analytics has already yielded promising results. Jagdale, Kolekara and Khot [20] explain assumption on computer-assisted diagnostics and prognostics system, which utilize multi-modal data e.g. computerized image processing and computerized clinical information like tissues and genetic data for making predictions and survival, as previously indicated. These initiatives diagnosis and provide prognostications for cancer patients using expression levels and other data analyzed using standard medical computing approaches. To anticipate whether patients react to pegylated interferon therapy, the authors investigated and presented a dataset array gene expression patterns data. Viral studies have begun to benefit from computer-assisted decision-making tools that have been modified using bioinformatics understanding. For example, based on expression patterns and hierarchical clustering of virus-host interactions, the authors pr; esent a computational strategy for finding the underlying principles of HIV-1 susceptibility in certain persons in an article. Another research uses a network-based analysis to present a unique way to identifying cirrhosis of the liver and hepatic disorders.

Table 1: Advantages and Disadvantages of the prevailing CDSSs and analysis of various areas of application

\begin{tabular}{|c|c|}
\hline $\begin{array}{l}\text { Area of } \\
\text { Application }\end{array}$ & ntages \\
\hline Tumor & $\begin{array}{l}\text { - Numerous cases of cancer include a } \\
\text { plethora of biochemical experiments and } \\
\text { data, which may be exploited to construct } \\
\text { robust CDSS }\end{array}$ \\
\hline
\end{tabular}

Radiology medication

\section{Cardiac medication}

- Despite the fact that just a few technologies have been implemented into diagnostic procedures, the operating practices have demonstrated a favorable influence on healthcare performance and costs.

- Because medical technology and injury are such time- and resource-sensitive parts of healthcare, computer-assisted solutions have a lot of promise in this field.

- Because heart disease is one of the most common causes of mortality, computerassisted decision-making systems might have a significant influence on global health.

- Whereas most cardiovascular-based cognitive management information systems have a significant rate of false positive, they frequently aid in the early detection of illness.

\section{Disadvantages}

- More work has to be done to incorporate early screening data from genetic and image-based modalities.

- It is necessary to create better technologies and methodologies for verifying the efficacy of present and future systems in this field.

- The majority of this field's research suffered from shortage of adequate data.

- The results of previous studies do not factor in knowledge of disease, damage, or complications when making decisions.

- Existing technologies' accuracy rates may not be enough for therapeutic trials.

- CDSSs have yet to tackle a wide range of diseases and traumas.

- Adequate verification of long-term and short-term results on the environment applying massive data is lacking.

- Typically, these technologies only include a part of the patient data that is accessible. To decrease false positive results, more diversity in sources of data may be necessary in the decision-making procedure.

- There is no rigorous vetting mechanism in place. Existing study claims must be put to the test in more realistic situations. 


\begin{tabular}{|c|c|c|}
\hline $\begin{array}{l}\text { Dental } \\
\text { treatment }\end{array}$ & $\begin{array}{l}\text { - Technological solutions have shown their } \\
\text { capacity to identify dental issues early on. } \\
\text { This kind of early identification aids in the } \\
\text { delivery of better preventative care. }\end{array}$ & $\begin{array}{l}\text { - Some of the methods used to capture data } \\
\text { for CDSS are quite costly, which prevents } \\
\text { their widespread adoption in actuality. }\end{array}$ \\
\hline
\end{tabular}

\section{Technical Issues and Implementation Barriers}

In a variety of domains, CDSS encounter major technological obstacles. Because biological systems are so complex, a therapeutic choice may rely on a vast amount of possibly relevant details. When suggesting a patient's treatment plan, an automated evidence-based biomedical framework, for illustration, might take into account the patient's condition, medical records, family background, and genetic factors, and also geographical and cultural patterns in disease incidence and authored medical studies on medicinal efficacy. Workflow compatibility is a major stumbling block for CDSS adoption in the clinic. Many healthcare support networks also generate a large number of notifications, which is a topic of disagreement. Aside from the irritation, doctors may stop paying attention to warning when technologies generate a large number of them (particularly those that do not need escalating). As a result, potentially vital signals may be overlooked.

\section{Maintenance}

The difficulties of combining the vast amount of clinical study that is released on a constant schedule are one of CDSS's major concerns. Clinical studies are conducted in thousands per year. At the moment, each of these researches must be reviewed by hand, appraised for empirical validation, and accurately put into the CDSS. The method of getting medical evidence and clinical skills and transferring them into a format that machines can modify to aid in clinical decision-making was described as "yet in its inception" in 2004. Nonetheless, it is more practical for a company to accomplish this systematically, even if inefficiently, than for every solitary physician to maintain pace with all of the research that is released. Incorporation of advanced data, in additional to being time-consuming, may be difficult to measure or integrate into an established decision-making schema, especially when clinical articles seem to contradict one another. Addressing these kinds of differences correctly is often the focus of clinical publications, which may take months to finish.

\section{Evaluation}

A CDSS must be able to demonstrate that it improves diagnosis process or outcomes in order to be useful. CDSS assessment is a procedure of evaluating its worth to develop the efficacy and reliability of the system. Since CDSSs provide a variety of objectives, there is no one statistic that can be applied to all of them. However, characteristics like reliability (with oneself and with professionals) may be found in a broad range of systems. A CDSS's assessment benchmark is determined by the program's objective: for instance, a diagnostics framework may be graded on the reliability and stability of its illness categorization (in contrast to the healthcare professional and other decision support frameworks). A higher rate of patients' development or significant fiscal remunerations for care professionals could be used to grade evidence-based healthcare systems.

\section{AUTHENTICATION AND STANDARDS FOR SUCCESS}

With so many clinical deployments of CDSS for a wide range of biomedical operations, it's critical to have a standard process for verifying, validating, and comparing multiple components and their results. Ksiądzyna [6], for example, investigated the effectiveness of 4 computer-based medical diagnostics used in internal medications: QMR, Meditel, Iliad, and Dxplain. At various publications, these technologies have been mentioned in various stages of development, appraisal, and implementation. The authors put these systems to the test on similar symptomatically demanding situations and evaluated their effectiveness using different predefined measuring scales. Assessments of productivity were based on a set of test criteria that were specified in advance, including instances that varied in substance and complexity. Another study looks at the effectiveness of a decision assistance system for anticoagulation medication. The authors present a clinical study of the network of interrelated patients in several facilities to see whether the CDSS is effective in stabilizing individuals on oral anticoagulant medication by beginning and sustaining therapy. The research states that the management information system increases the quality of thromboprophylaxis, both during lengthy therapies and in beginning, unpredictable periods of medication, relies on empirical evaluation of performance parameters.

Several articles also go through the different factors that must be addressed in order to create and implement a CDSS successfully. In this vein, investigations look at the research on CDSS, focusing on performance standards. The author of the report says that, as a result of the early success, there is widespread excitement among doctors and academics for the possibility of CDSS to enhance quality of healthcare. Nevertheless, there is a scarcity of literature knowledge of 
such methods, particularly from the viewpoint of non-physicians, as well as why some diagnostic assistance systems may not be useful. Conversely, studies look at the impact of CDSS on physicians' perspectives; specifically if they appreciate their colleagues' ideas when they differ from their own. They argue that doctors are prone to trusting advice from CDSS emphasizing the significance of quality assurance and validation. According to studies, having a dependable and actual methodology of actual composite to assess the effect of the diagnostics decision provision on the eminence of biomedical is fundamental. The scoring approaches could be utilized to evaluate performance indicators in various kinds of studies that involve computer-assisted assessment methods. The many methodologies, aims, and features of computeraided medical procedures are discussed by authors in [22]. From the standpoints of health informatics and computer programming, the authors examine workflow implementation difficulties and software obstacles. Senarath [23] presented a report that identifies four critical aspects of diagnostic assistance material that are linked to the efficacy of CDSS. The research conducts a comprehensive evaluation of documented trials in order to determine the features of the methodology used and the practicalities of studies evaluating the effectiveness of CDSS [24-28].

\section{CONCLUSION AND FUTURE DIRECTIONS}

With the large quantity of medical computing approaches applied as computer-assisted diagnostic and CDSS, as well as the massive number of studies in this subject, such systems will ultimately become an integral branch of healthcare. Clinical issues are growing more complicated and complexes, as well as the algorithms are becoming able to address them. These computer-assisted techniques may become much more trustworthy by implementing systematic authentication and authorization procedures, which will enhance the reliability of diagnosis choices and minimize variation among doctors' perspectives. These technologies' unique characteristics enable caretakers and academics to get overview of current methodological problems in methods that were previously unattainable. Furthermore, integrating information produced from healthcare records with many modalities to deliver more powerful diagnosis and effective plan ideas is becoming increasingly useful. The present convergence of biomedical computing and informatics approaches will hasten the development of a novel phase of system-biologic CDSS, which will analyze and integrate data from sequence genetics, signals and pictures, and demography, among other sources. Such algorithms will be able to produce significantly more detailed and individualized suggestions thanks to these and other types of patient records. Applying developments in computational tools and approaches to such systems might assist with issues including imbalanced datasets of outputs to certain kinds of data, sensitivity to inadequate or incomplete data, and the availability of contradicting data from several sources. These advancements in computational approaches may also increase the quality of data obtained via feature extraction process selection - a crucial step before classification and aggregation. Whereas computerised predictive and therapeutic decision support initiatives have indicated to be applicable in the field of medicine, which they will eventually become more useful as they improve to analyze and incorporate more and larger forms of patient records.

\section{References}

[1]. G. Rzevski, "Computer-aided design of control systems", Computer-Aided Design, vol. 11, no. 6, p. 363, 1979. Available: 10.1016/00104485(79)90043-5.

[2]. A. Ciapponi, "Does a mobile clinical decision-support system (CDSS) help improve the quality of primary health care?", Cochrane Clinical Answers, 2021. Available: 10.1002/cca.3745.

[3]. S. Ji, R. Smith, T. Huynh and K. Najarian, "A comparative analysis of multi-level computer-assisted decision making systems for traumatic injuries", BMC Medical Informatics and Decision Making, vol. 9, no. 1, 2009. Available: 10.1186/1472-6947-9-2.

[4]. L. Pinheiro, G. Candore, C. Zaccaria, J. Slattery and P. Arlett, "An algorithm to detect unexpected increases in frequency of reports of adverse events in EudraVigilance", Pharmacoepidemiology and Drug Safety, vol. 27, no. 1, pp. 38-45, 2017. Available: 10.1002/pds.4344.

[5]. "Of current interest: Radio-electrocardiograph system for electrocardiograms while patients exercise", Electrical Engineering, vol. 80, no. 7 , pp. 556-557, 1961. Available: 10.1109/ee.1961.6433350.

[6]. L. Arknæs-Pedersen, "Method and an apparatus for processing an auscultation signal", The Journal of the Acoustical Society of America, vol 120, no. 2, p. 583, 2006. Available: 10.1121/1.2336707.

[7]. N. Sánchez-Pi, J. Carbó and J. Molina, "A knowledge-based system approach for a context-aware system", Knowledge-Based Systems, vol. 27, pp. 1-17, 2012. Available: 10.1016/j.knosys.2011.08.017.

[8]. S. Kositbowornchai, S. Siriteptawee, S. Plermkamon, S. Bureerat and D. Chetchotsak, "An Artificial Neural Network for Detection of Simulated Dental Caries", International Journal of Computer Assisted Radiology and Surgery, vol. 1, no. 2, pp. 91-96, 2006. Available: 10.1007/s11548-006-0040-x.

[9]. M. Moinet, G. Mandil and P. Serre, "Defining tools to address over-constrained geometric problems in Computer Aided Design", ComputerAided Design, vol. 48, pp. 42-52, 2014. Available: 10.1016/j.cad.2013.11.002.

[10]. A. Azami, "Credit Derivatives: CDSs and Value's Firms; Effect on Financial Statements", SSRN Electronic Journal, 2011. Available: $10.2139 /$ ssrn. 1940716.

[11]. A. Segev, "Integrating computer vision with web-based knowledge for medical diagnostic assistance", Expert Systems, vol. 27, no. 4, pp. 247258, 2010. Available: 10.1111/j.1468-0394.2010.00520.x. 
[12]. H. Atabakhsh, "A survey of constraint based scheduling systems using an artificial intelligence approach", Artificial Intelligence in Engineering, vol. 6, no. 2, pp. 58-73, 1991. Available: 10.1016/0954-1810(91)90001-5.

[13]. G. Campanella and R. Ribeiro, "A framework for dynamic multiple-criteria decision making", Decision Support Systems, vol. 52, no. 1, pp. 52-60, 2011. Available: 10.1016/j.dss.2011.05.003.

[14]. A. Bagheri and M. Mardaneh, "Fuzzy logic-based technique for enhancement of kalman filter based PLL", Journal of Intelligent \& Fuzzy Systems, vol. 28, no. 3, pp. 1371-1383, 2015. Available: 10.3233/ifs-141421.

[15]. J. Cockx, K. Denolf, B. Vanhoof and R. Stahl, "SPRINT: A Tool to Generate Concurrent Transaction-Level Models from Sequential Code", EURASIP Journal on Advances in Signal Processing, vol. 2007, no. 1, 2007. Available: 10.1155/2007/75373.

[16]. Y. Malla, "A Machine Learning Approach for Early Prediction of Breast Cancer", International Journal Of Engineering And Computer Science, 2017. Available: 10.18535/ijecs/v6i5.31.

[17]. M. Yang, S. Nazir, Q. Xu and S. Ali, "Deep Learning Algorithms and Multicriteria Decision-Making Used in Big Data: A Systematic Literature Review", Complexity, vol. 2020, pp. 1-18, 2020. Available: 10.1155/2020/2836064.

[18]. J. Zimmerman and J. Rothmeier, "History and future of MUMPS in medical computing", Medical Informatics, vol. 4, no. 1, pp. 5-11, 1979. Available: 10.3109/14639237909044355.

[19]. J. Gero, "Special issue: Artificial intelligence in computer-aided design: Progress and prognosis", Computer-Aided Design, vol. 28, no. 3, pp. 153-154, 1996. Available: 10.1016/0010-4485(96)86821-7.

[20]. S. Jagdale, M. Kolekara and U. Khot, "Smart Sensing Using Bayesian Network for Computer Aided Diagnostic Systems", Procedia Computer Science, vol. 45, pp. 762-769, 2015. Available: 10.1016/j.procs.2015.03.150.

[21]. D. Ksiądzyna, "Drug-induced acute pancreatitis related to medications commonly used in gastroenterology", European Journal of Internal Medicine, vol. 22, no. 1, pp. 20-25, 2011. Available: 10.1016/j.ejim.2010.09.004.

[22]. "Computer Aided Detection of Polyps During Colonoscopy Procedures", Case Medical Research, 2019. Available: 10.31525/ct1nct04195646.

[23]. S. Senarath, "Securitization and Credit Default Swaps (CDSs): Towards Diagnostic of the Fundamental Problem and Suggested Solutions", SSRN Electronic Journal, 2014. Available: 10.2139/ssrn.2478313.

[24]. A. Haldorai and A. Ramu, "Canonical Correlation Analysis Based Hyper Basis Feedforward Neural Network Classification for Urban Sustainability,” Neural Processing Letters, Aug. 2020. doi:10.1007/s11063-020-10327-3

[25]. D. Devikanniga, A. Ramu, and A. Haldorai, "Efficient Diagnosis of Liver Disease using Support Vector Machine Optimized with Crows Search Algorithm," EAI Endorsed Transactions on Energy Web, p. 164177, Jul. 2018. doi:10.4108/eai.13-7-2018.164177

[26]. H. Anandakumar and K. Umamaheswari, "Supervised machine learning techniques in cognitive radio networks during cooperative spectrum handovers," Cluster Computing, vol. 20, no. 2, pp. 1505-1515, Mar. 2017

[27]. H. Anandakumar and K. Umamaheswari, "A bio-inspired swarm intelligence technique for social aware cognitive radio handovers," Computers \& Electrical Engineering, vol. 71, pp. 925-937, Oct. 2018. doi:10.1016/j.compeleceng.2017.09.016

[28]. R. Arulmurugan and H. Anandakumar, "Early Detection of Lung Cancer Using Wavelet Feature Descriptor and Feed Forward Back Propagation Neural Networks Classifier," Lecture Notes in Computational Vision and Biomechanics, pp. 103-110, 2018. doi:10.1007/978-3319-71767-8_9 